\author{
Hantzsch, C A \\ Ueber einige Diatomaceen \\ aus dem ostindischen Archipel
}




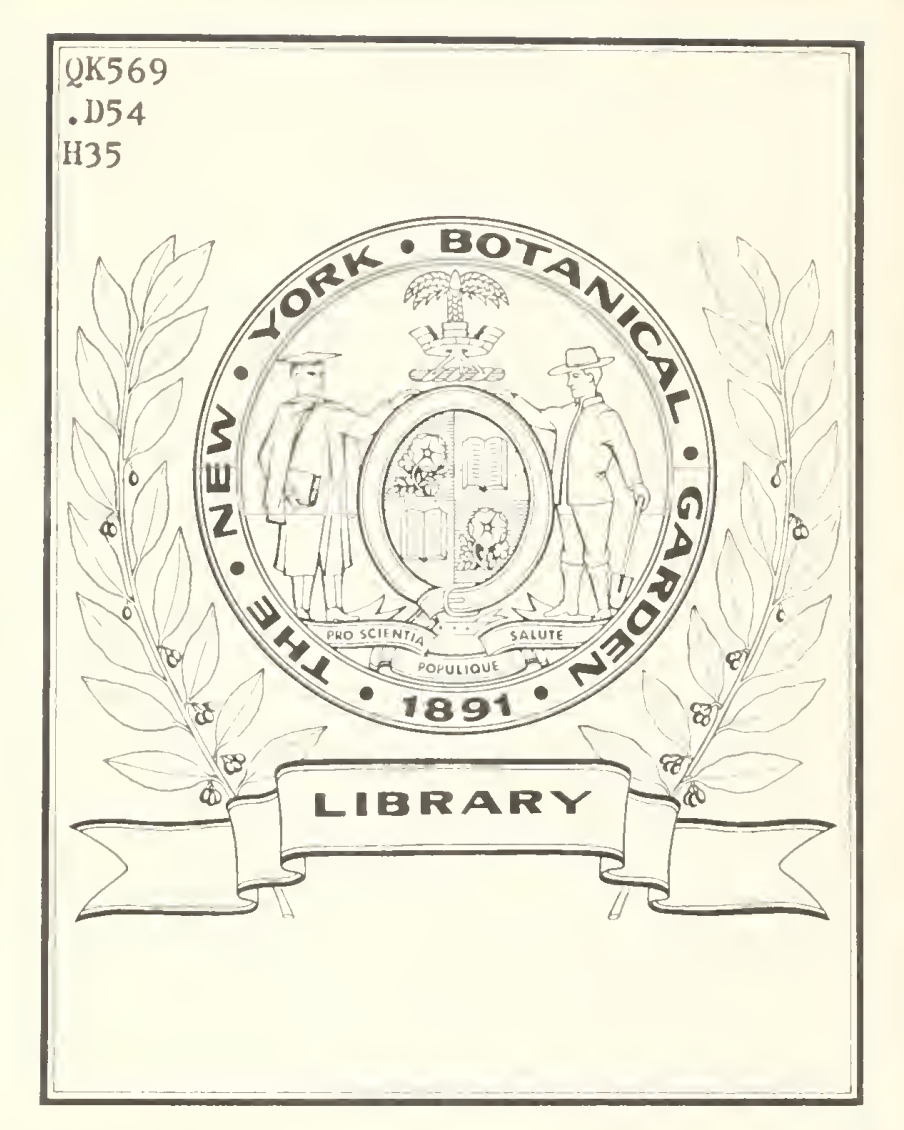








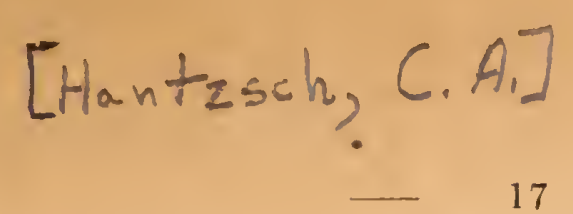

\title{
Ueber einige Diatomaceen aus dem ostindischen Archipel.
}

\author{
Hierzu 'afel V. und VI. A.
}

Unter Nr. 1403 in Rabl. Algen Enropas's habe ich ein Prïparat geliefert. Welches reicl an interessanten Diatomaceen ist. Dieselben wurden durch Absclılenmen und I'rüpariren (Kochen mit Salyetersäure und chlorsaurem Kali) ron Algenresten und Meeresschlamm aus dem ostindischen Arelipel gewomen.

Die darin enthaltenen Arten sind:

Grammatoplıora oceanica Elırb. (Ehrb. Nicrogeologie etc.)

Grammat. macilenta Sm. rar.?

Grammat. subtilis und subtilissima Aut. ?

Rhabdonema adriaticum Küt $z$, sehr zarte und schlanke Form. (Ktz. Bacill. und Smith. Brit. Diat. T. 38. die drei untersten Figuren). Man möclite dieselbe wolıl für eine eigene Art halten, jedoch lässt sich in Bau durchaus kein characteristischer Unterschied finden; namentlich sind deshalb die grössten Fxemplare dieses Rlıabd. mit den kleinsten Exemplaren des gewölnlichen Rlıabd. adr. aus dem mittelländischen Neere etc. zu vergleichen, welches ich gleichzeitig mit cingeliefert liabe.

Climacosira mirifica Grunow (Grunow, die österr. Diat.)

(Rlabdonema mirificum Sm.)

- Climacosphenia indica Htzsch. Fig. 1.

- Synedra pulcherrima Htzsch. Fig. 2.

- Synedra formosa Htzsch. Fig. 3.

- Toxarium rostratum Htzsch. Fig. 4.

Naricula Lyra Fhrb. in dir. Varietäten.

$"$ splendida Greg. (Ld. Micr. J. 1858).

, Hennedyi Smitlı. (Li. Micr. J. 1856).

Hierzu woll auch Nav. clarata Greg, ebendaselbst.

" forcipata Grer. (Micr. J. 1859). (N. Smithii rar. suborbicularis Greg. in

Greg. on New forms of mar. Diat. T. 17.)

, Liber Ehrb.

Stauroptera spec.?

Plagiogramma spec.?

Mastogloia apiculata Thw. (Sm. Brt. Diat.)

, $\quad$ interrupta Htzscl. Fig. 5.

". quinquecostata Grunow. var. Fig. 6. (Grun. über einige neue Algen etc.)

Nitzschia panduriformis Greg. Fig. 7. (Greg. New forms etc. T. VI. 102.)

.. Formica Htzscl. Fig. 8.

Tryblionella constricta Greg. (Greg. New forms.)

Epithemia MLusculus Ǩ́tz. (?) Sm. Brt. Diat.

Euplyyllodium spathulatum (Aut.?) Mier. J. 1854. 
Surirella fastuosa Elırb. sehr kleiı.

Eupodiscus minutus IItzsch. Fig. 9,

Coscinodiscus (?) punctulatus Freg. (Greg. T. II. 46.)

Podosira laevis Greg. (Micr. J. 1859.)

Cocconeis fimbliata Brightw. (Micr. J. 1859.) Die Zahl der Bogen an der inner'n Platte ist veränderlich; man findet deren an verschiedenen Exemplaren an jeder Hälfte der Platte 3 his 6 .

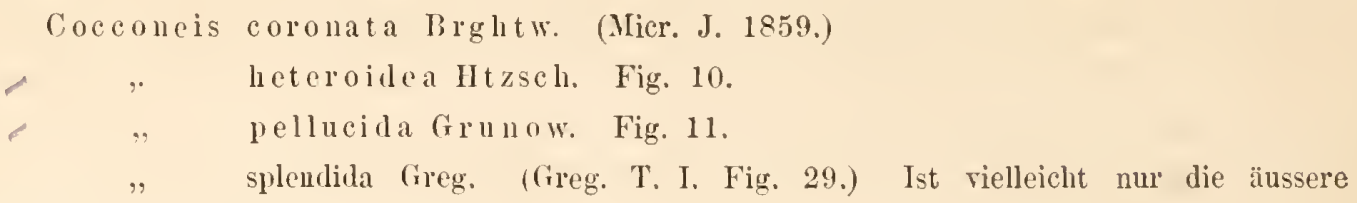

Platte ron Coce, timbriata orler coronata?

Cocconeis distans Greg.? (Micr. J. 1855.)

$\because \quad$ regina C. Johnst. (Micr. J. 1859.)

(liem. wie bei Coce. splendida.)

Ansserlem noch einige kleine Diatomaceen und unhestimmbare Bruchstïcke, sowie allerhand Nadeln und Kiystalle aus Schwämmen.

In Nachfolgenden gebe ich nïheren Nachweis über die noch nicht bekannten und nicht sicher bekamten Arten. Die Grössen sind in Millimetres ausgedrückit.

1. Climacosphenia indica Htzscl. Fig. 1.

$$
\begin{aligned}
& \text { Länge }=0,330-0,480 . \\
& \text { Breite oben }=0,030-0,036 \text {. } \\
& \text { unten }=0,010-0,012 .
\end{aligned}
$$

Aeussere Platte (c) schwach gelhlich mit 2 starken, nach oben divergirenden, nicht ganz geradlinigen Längsrippen, welche oben sich gegeneinander krümmen, jedoch nicht zusammentreffen, nach unten nach und nach verschwinden. Zarte Querstreifung, durchaus parallel.

Innere Platte (a, b) mit grossen Oeftuungen, welche nach oben hin fast rechteckig, nach unten hin oval his kreisrund werden. In mntern Theile der Platte befindet sich zwischen den Oefinungen, welche lier im Verhältniss zm Grösse ctwas weitläutiger stelen. cine (mitunter auch zwei bis drei) Verdicliungen, welche nach dem obern Theile der Platte zu immer mudentlicher werden und endlich, während gleichzeitig die scheidewände immer selnnaler werden, ganz verschwinden. Die Grösse und das Verhältniss der Oeffnungen und Verdickungen ist sehr sehwankend (a. und $a^{\prime}$ ).

Ausgebildete Nebenseite (d) ebenso breit wie die Hauptseite, ebenfalls zart quergestreift, nur ganz oben in zwei diagonal sich krenzente streifungen übergehend.

Die beschaffenheit der stiele komnte nicht beobachtet werden.

Vorliegende Climacosphenia lat viel Aehnlichlieit mit der (C. catena (Aut.?) im Id. Micr. J. 1851 und Cl. elongata Bailey (Notes on New Species and Lokalities of Micr. Org.) Erstere ist aber im Verhältuiss zur Breite viel kïrzer und hat wie letztere lieine Verdickmgen zwisehen den Oeffumngen; diese sind lurehaus rechteckig. Bei beiden ist die äussere Platte nicht beriicksichtigt worden.

Grunow (die österr. Diat.) giebt eine 7achnung, welche rorliegender Art fast entsprechen 
würde und stellt sie fraglich als $\mathrm{Cl}$. elongata Bail auf. Aus angegebencn Grïnden kann man jedoch keine der bisherigen Beschreibungen und Abbildungen, dic freilich möglichcrweise unvollständig oder ungenau sind, hierauf bezichen.

2. Syncdra pulcherima Htzseh. Fig. 2 .

$$
\begin{aligned}
& \text { Längc }=0,240-0,360 . \\
& \text { Breitc }=0,017-0,029 .
\end{aligned}
$$

Diese ansgezeichncte Art ist, wie dic folgende, achthantig und crscheint blau bis violett. Enden kopfförmig erweitert, rund oder stmmpf zngespitzt. Mitmuter sind dic Köpfe nur angedeutet, niemals aber tritt eine Verschmälerung am Ende cin. Dic Kianten verlaufen am Kö̈rper genau parallel, erweitern sich am Kopfe je nach desscn Umfang und laufen in der Spitze zusammen. In ganzen Unfange starke Querstreifung. Dic Streifung, sowohl bei dieser als auch der folgenden Art ist eine doplette, eịe auf der inneren und eine anf der äusseren Fläche des Panzers. Bei einer Vergrösserung von $400 / 1$ und mehr zeigt sich, wenn man die Platte etwas muter den Phoeus cinstellt, eine wirkliche Strcifung, jeder Streifen als einc sehr scharfe Doppellinie (s. Fig. 3. c.), dagegen zeigen sich bei hoher Einstcllung Streifen aus dentlichen, kriftigen Punkten bestchend s. Fig. 2. е.) - Ian könnte hicr eine optische Tänsehnng vermuthen, der Unterschicd der bciden Einstcllungen ist jedoch so bedeutend, dass cine anderc Deutung als obige nicht gut möglich ist.

3. Sylledra formosa Htzsel.. Fig. 3.

$$
\begin{aligned}
& \text { Länge }=0,214-0,310, \\
& \text { Breite }=0,017-0.022 .
\end{aligned}
$$

Achtkantig wie vorige. Im Uebrigen fast ganz mit Synedra superba (Kütz.) Sm. übereimstimmend. Die Umrissc (Kanten) sind jedoch nicht so regelnässig, sondern etwas uneben. Die Mitte des Körpers ist am dicksten, nach den Enden zu ganz unbedcutend. meist (Fig. $3^{c}$ ) kurz vor dem Ende aber plötzlich etwas mehr rerschmälert. Starkc Querstreifung. Erscleint ebenfalls blau und hellviolett. Die Schlcimstielc konnten nicht beobachtet werden.

So ähulich die S. supcrba Sm: ist, so ist diese jedoeh nach Smith's Zeiehnung nur vierseitig mit verbroehenen Kanten, daher nicht dieselbe.

Ferner giebt Grunow (a. a. 0.) eine sehr älmliche Zeichnung, aueh achtkantig, als: Synedra dalmatica Kitz., lässt es jedoeh fraglich. da sic nach Kütz. nieht genau zu bcstimmen ist. Dieselbe Art giebt Pritchard (in Hist. of Infusoria T. VIII. Fig. 3) als Synedra robusta. Sie ist breiter als S. formosa und an den Enden nicht rerjüngt.

4. Toxarium rostratum Htzsch. Fig. 4 .

$$
\begin{aligned}
& \text { Länge }=0,380-0,530, \\
& \text { Breite }=0,012-0,020 \text { in der Mitte. }
\end{aligned}
$$

Dicke der Schnäbcl ea. 0,005.

Von der Gattung Toxarium Bailey ist bis jetzt nur eine Art bekannt: T. undulatum Bailey (in Bailey, on New Spccies etc.), wobei als Synonym: Synedra undulata B. (Greg.) angeführt wird.

Tox. rostratum ist dem Tox. undul. sehr ähnlieh, die Schnäbel jedoch nicht wellcnförmig gebogen; es ist nicht so schlank, der angeschwollene Mittelkörper grösser und breiter. Die Ansehwellung der Schnabelenden sehr reränderlieh, kaum bemerkbar bis auf das Doppelte der Breite des Schnabels. 


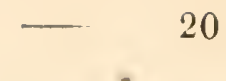

Die Oberfläche ist mit zicmlich weitlüufig gestellten Punkten rerschen. meist in unrcgelmässig-diagonaler, oft in ganz unregchmässiger Anordnung, welche noch dadnrclı rermehrt wird, dass hier und da kleine Lücken in der Punktirung sich befinden (Fig. 4 c.) Eine parallele Streifung habe icl nie bemerkt. Punktreilıen am Rande oft etwas kräftiger, als die Punkte der Fläche, - Panzer robust, etwas bräunlich.

Grunow stellt Toxarium als Unterabtheilung unter Synedra, indem er dabei anführt: ¿Querstreifen dic Mittellinie nicht elreichend." Diese Abtheilung würde also diejenigen SynerlraArten enthalten, welche die Form von Toxariun, aber die Streifung von Synedra haben; dergleichen Exemplare sind mir jedoch noch nicht vorgekommen. Toxarimm als Gattung dagegen würde sich durch die zerstreut orler diagonal gestellten Punktrcihen miterscheiden. Bailey giebt zwar für sein T. undul. Querstreifung an, aus der Abbillung geht dies jedoch nicht hervor, dicse zeigt rielmelır eine umrcgehmässige Punktirung. Nach Fxcmplaren von zwei rerschiedenen Orten erkeme ich die Bailey'sche Art als bestimmt zu Toxarium gehörig. Deutlich kann man die Structur nur am Mittelkörper erkennen, da die Schnälıel nnd die Enden schmal und stielrund sind, wodurch lcicht optische Täuschung entsteht.

5. Mastogloia interupta Htzscl. Fig. 5.

$$
\begin{aligned}
& \text { Länge }=\text { ca. } 0,072 \text {. } \\
& \text { Breite }=\text { ca. 0,022. }
\end{aligned}
$$

Form lanzettlich-rhomboidisch, indem die Seiten ron der Spitze nach der Mitte fast gerade verlaufen und dort fast eimen stumpfen Winkel bilden; am Ende plötzlich eingezogen mit eimer aufgesctzten, stumpflichen Spitze. Aeussere PIatte am Rande quer gestreift, die Streifung mit einer nnregelmässigen Linie eingefasst; diese Zeichnung ist ncben der Mitte am breitesten. Innere Platte, jener Einfassung entsprechend mit einer Rippe, welche an jencn breitcren Stellen denselben entsprechend mit eincr Anschwellung mit 3 bis 5 starken Querripen rersehen ist. Mittelrippe mmregclmässig hin - und lergchogen mit lïnglichem Mittelknoten.

Iclı füge unter Fig. 5c. noch die Zeichnung einer Platte bei, welche wahrscheinlich noch als äusserste Platte zu dieser Mastogloỉa gehört; sie stimmt in Form und Grösse genau damit ïberein und ist an und für sich zu keiner bestehcnden fattung zu bringen, wegen zu sparsamen Vorlandenseins in der Masse liess sich dies jedoch nicht bestimmt ermittelı.

6. Mastogloia quinquecostata Grunow rar. (Glum. Nene mul mingenïgend gek. Algen). Fig. 6 .

Weicht ctwas in Form und Zeichnumg ron Grunow's Figur ab, wurle jedoch ron Herm Grunow fïr seine M. quinquec, bestimmt. Sie ist kleincr als erstere. Enden nicht vorgezogen nnd die weitlänfigeren Quer-Rippen am Rande nit einer unregelmässigen Einfassmng. die ein Stück ror dem Ende sich ganz an den Rand anlegt. Die Lïngsrippen mïnden ein Stiick ror der Spitzc in die Mittelripne cin. Mittelknoten länglich. Aeussere Platte halse ich nicht anffinden kömnen.

7. Nitzsclia panduriformis Greg. (Greg., on new forms of marine Diatom.) Fig. 7.

I)ieselhe ist sehr vielgestaltig und findet sich in dem angefülırten Präparate sowohl in der ron Greg. gezcichmeten Form, als anch in selır abweichenden ror; Panzer brïunlich, rohnst.

Gregory setzt ein Fragezcichen zur Gattung, jecloch lassen die Exemplare sowohl, als die Zcichunng G.'s krinen Zweifel darïber zu. 
8. Nitzschia Formica Htzsch. Fig. 8.

$$
\begin{aligned}
\text { Iänge }= & \text { ea. } 0,100 \\
\text { Breite }= & \text { ca. } 0,01+\text { in ler Mitte. } \\
& \text { ca. } 0,020 \text { an den breitesten Stellen. }
\end{aligned}
$$

Stumpf lanzettlich, in der Mitte mit starker, germndeter Winschnürung. P'atten selır gewölbt. Querstreifung selu denthch. Zwei Reihen längliche, sehr dentliche Punkte am Rande. Panzer briannlich, rohust.

Hierbei bemerke ich, dass ich nach wiederlsolter. Untersnchmeg der von mir in Rabh. Alg. Sachs. N1. 984 gelieferten Nitzschia 'Tryblionella m. dieselbe nicht für identisch mit der irrthïmlicl als Synonym citirten Tryblionella gracilis Sm. halten kam. Grunow hat mu erstere „Tryblionella Ilantzschiana“ benannt, dagegen die letztere mit Recht als ... ur irella gracilis* bezeichnet.

Arten von Tryblionclla, die genan mit Smith's Beschreibung ïbereinstimmen, sind mir noch nicht vorgekommen, noch habe ich dies von Anderen erfalnen. Dagegen scheinen ron manchen Antoren Nitzschia-Alten mit mehrfachen Punktreihen als Tryblionella betrachtet worlen zu sein.

9. Fupodiscus minutus Htzsch. Fig. 9.

$$
\text { Durchmesser }=0,043-0,057 \text {. }
$$

Platten meist gelblich. flach. unr am Rande eingebogen; 12-16 strahlig. Die Strahlen bestehen ans regelmässig gestellten Punkten. welche nicht ganz bis zm Mitte reichen. dic mit 3-5 mmegelmässig gestellten Punkten bezeichnet ist. In den durch die Strahlen gebildeten Feldern sind die Pmnkte von der Mitte aus eimreihig, uach dem Rande zu breiter grupluirt, aber nicht in vollkommen regelmässige Fignren gestellt. Ein kleiner Fortsatz (Iörnchen) ganz an Rande.

10. Cocconeis leteroidea Htzsch. Fig. 10.

$$
\begin{aligned}
& \text { Iänge }=0,043-0.065 . \\
& \text { Breite }=0,029-0,048 .
\end{aligned}
$$

Form verschieden, schmal bis breit oval. Iängsleisten der innern Platte jederseits zu 2-4 ron der Mitte abstehend, oben und unten verdünnt nud fast zusammenlanfend, eine lanzettliche Figur billend, deren Axe nicht mit der Hautaxe zusammenfällt, sondern diese kreuzt. Ansserdem oft noch mit einer Ringleiste am Rande. Mittellinie der äussern Platte S förnig, ebenfalls die Hauptaxe schrïg kreuzend nnd in cine Ringleiste einmïndend. Mittelknoten quer verlängert, mitunter in eine wirkliche Qnerleiste bis zmm Rande verlanfend.

11. Cocconeis pellucida A. Grunow. Fig. 11.

$$
\begin{aligned}
& \text { Länge }=0,055-0,090 . \\
& \text { Breite }=0,036-0,072 .
\end{aligned}
$$

Meist breit oval, mitunter unmerklich zugespitzt.

Längsleisten der innern Platte oben nicht zusammenlaufend, sondern in ziemlich gleichen Abstïnden in eine vom Rande abstehende Ringleiste einmündend. Die beiden der Mitte zunächst liegenden fast parallel, einen breiten Mittelknoten einschliessend; die folgenden bogenförmig, daher die Zwischenrüume in der Mitte erweitert: sämmtliche I_eisten mmregelmässig-wellig. Mittellinie der änsseren Platte gerade mit länglichem Mittelknoten. Ringleiste nur an den Berührungspunkten der Mittellinie deutlich, nach den Seiten zu oft ganz rerschwindend, mitnnter schwach angedeutet. 
Aeussere Platte oft gelblich. Doppelt diagonale Streifung an Rande bei grossen Exemplaren deutlich, nacli der Mitte verschwindend.

Letztere beilen Arten sind von den bisher bekannten Cocconeis so abweichend, dass man sie wohl als eine neue Gattung betrachten könnte, wovon ich jedoch vor der Hand absehe. Mit Recht lıat auch Grunow auf die nahe Verwandtschaft einiger grösseren marinen CocconeisArten mit Mastogloia hingewiesen. Es lürften sonach überhaupt noch weitere gründliche Untersuchungen abzuwarten sein.

\section{Erläuterung der Tab. Y.:}

1. Climacospluenia indica Htzscl.

a. ganze Hauptseite,

$a^{\prime}$ u. b. Bruchstücken,

c. äussere Platte,

d. Nebenseite nach vollendeter Theilung.

2. Synedra puleherrima Htzsch.

3. , formosa Htzsch.

4. Toxarium rostratum Htzsch.

Erläuterung der Taf. VI. A.:

5. Mastogloia interrupta Htzsch.

5 c. Do.? äussere Platte?

6. Mastogloia quinquecostata Grunow rar.

7. Nitzschia panduriformis Greg. var.

8. Tryblionella Formica Htzsch.

9. Eupodiscus minutus "

10. Cocconeis heteroidea ",

a. innere und äussere Platte,

$b^{\prime}, b^{\prime \prime}, b^{\prime \prime}$ äussere Platten,

c', c" innere Platten.

11. Cocconeis pellucida Grunor.

a. innere Platten,

b. üussere Platten.

(Sämmtliche Figuren $=420 / 1$ mit Ausnahme von $3 \mathrm{c}$ und $4 \mathrm{~d}=700 / 1)$.

Dresden, October 1862.

C. A. Hantzsch. 


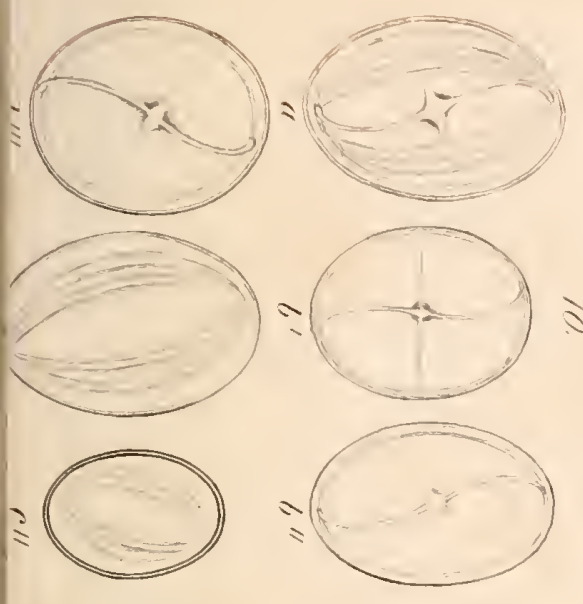

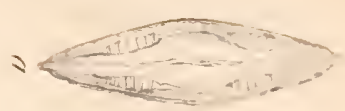

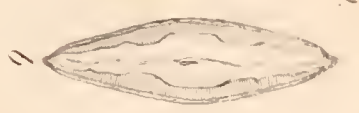

ते
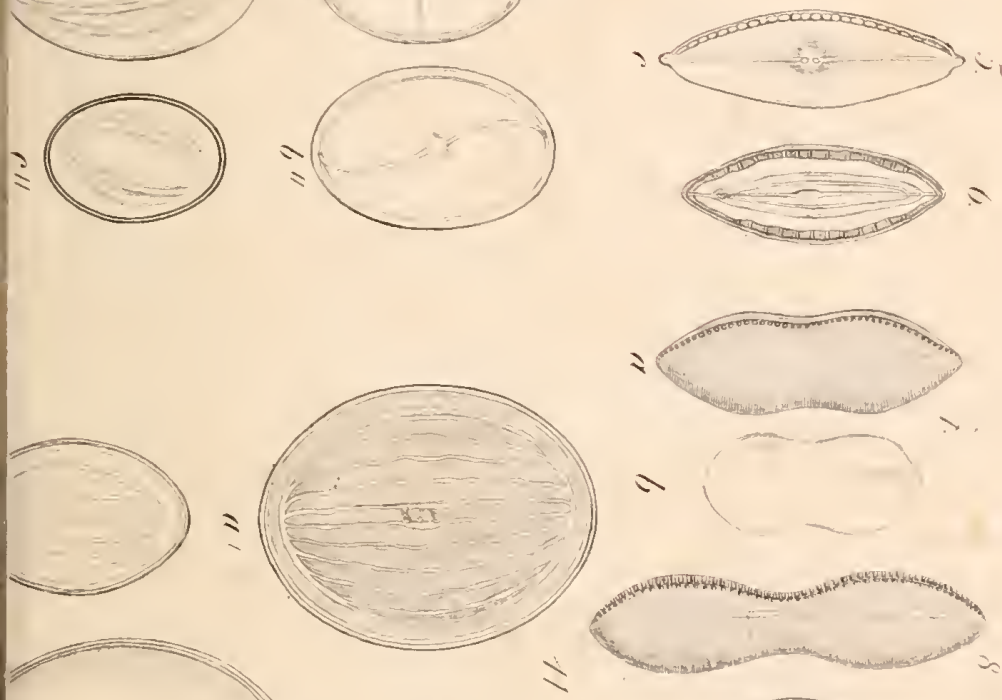

N

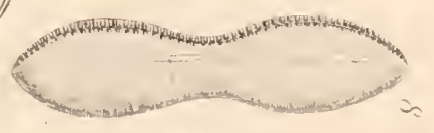

$\rightarrow$
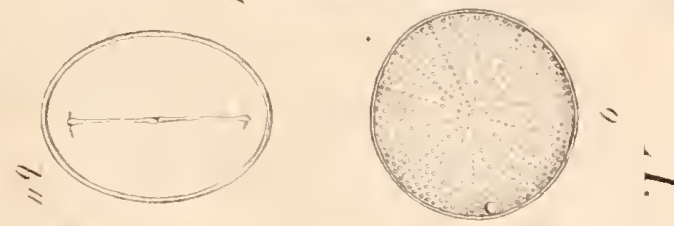

$\Sigma$ 

T"Il. I:

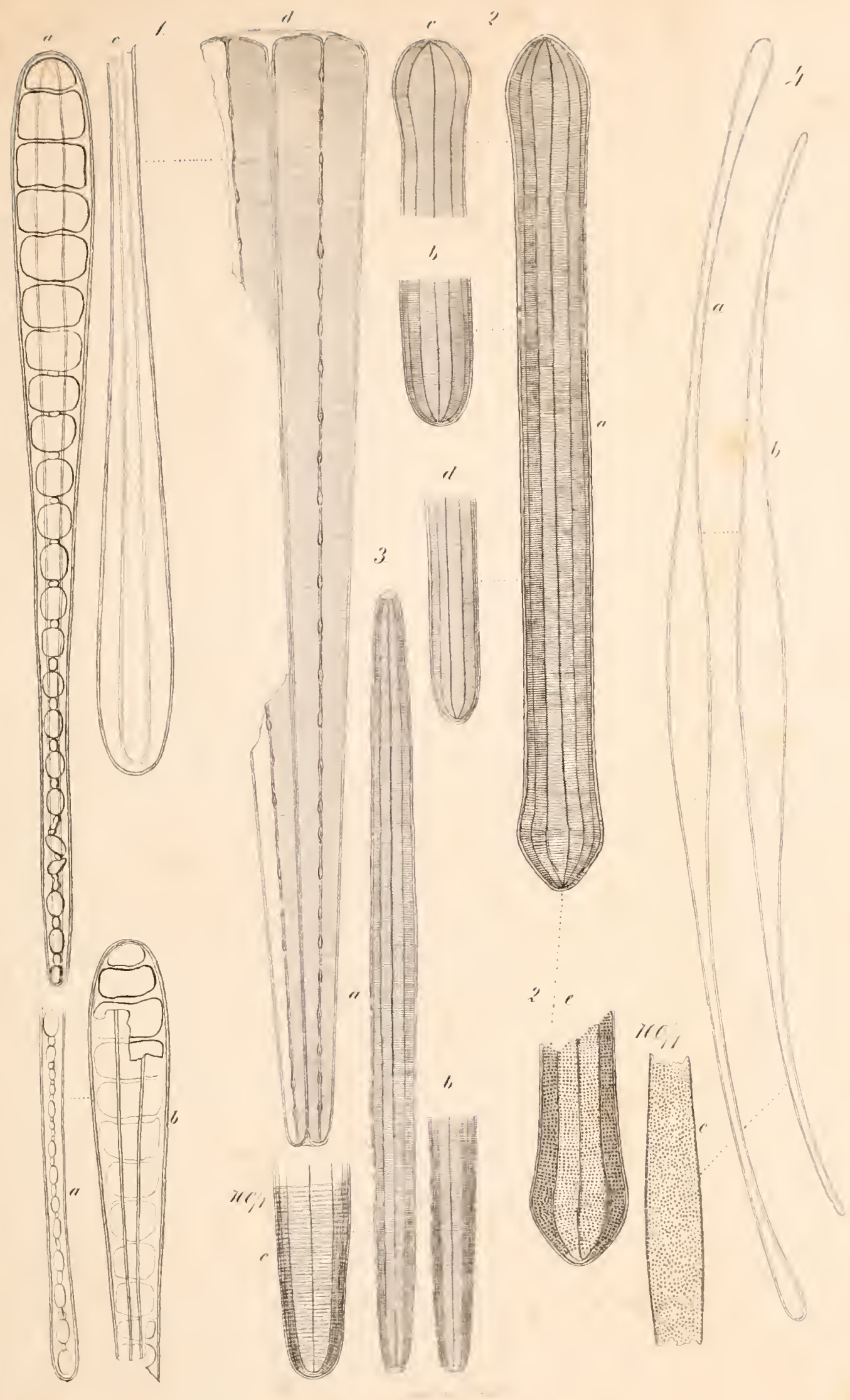










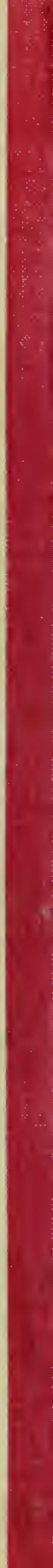

